\title{
Jornalismo para a paz: conceitos e reflexões ${ }^{1}$
}

\section{Raquel Cabral e Jorge Salhani}

\section{Resumo}

A violência cultural é legitimada à medida que ela predomina nos discursos sociais e nos produtos culturais. Nesse contexto, os estudos para a paz (Peace Studies) surgem a partir de uma perspectiva multi e interdisciplinar a fim de visibilizar as estruturas da violência, propor alternativas para a construção de uma cultura de paz e consolidação da paz positiva. Este artigo, sob o guarda-chuva dos estudos para a paz, apresenta a perspectiva do jornalismo para a paz (Peace Journalism) como reivindicação de um novo fazer jornalístico. Por meio de pesquisa bibliográfica e exploratória, refletimos sobre a viabilidade do jornalismo para a paz e apresentamos duas possibilidades distintas para sua utilização, uma de caráter prático e profissional e outra de caráter metodológico.

\section{Palavras-Chave}

Cultura de paz. Jornalismo para a paz. Paz. Violência.

\section{Raquel Cabral | raquelc@faac.unesp.br}

Doutora em Comunicação Institucional pela Universitat Jaume IEspanha. Professora do Programa de Pós-Graduação em Comunicação da UNESP - Universidade Estadual Paulista, Bauru-SP, Brasil.

Jorge Salhani I jorge.salhani@hotmail.com Mestrando do Programa de Pós-Graduação em Comunicação da UNESP - Universidade Estadual Paulista, Bauru - SP, Brasil.

\section{Os Peace Studies: a paz como objeto de estudos}

0 uso da violência é naturalizado à medida que a sociedade se omite ao adotar políticas alternativas de mediação e transformação de conflitos, o que aprofunda as desigualdades e promove injustiças visíveis (VALESAN, 2015). Johan Galtung (2003) afirma que a violência direta e a violência estrutural são institucionalizadas pelos aspectos de uma cultura, o que dá origem à violência cultural.

Anteriormente a conceber a violência cultural como um conceito complexo e fundamental para entender a sociedade atual, o autor apresenta diferentes tipologias para a violência: primeiramente, ele distingue a violência física da psicológica; ao fazer isto, extingue-se a ideia de que a violência é reservada apenas ao ato físico.

Galtung propõe, como primeira definição, a violência direta, na qual, segundo ele, é possível identificar a vítima e 0 agressor. A mais importante indagação feita por Galtung se concentra na existência ou inexistência de um 
indivíduo violento. A partir disto, o autor defende que existe violência mesmo onde não há atores específicos que a cometam. Este tipo de violência é definido como indireta ou estrutural, e é construído socialmente sob a forma de poderes desiguais e, consequentemente, oportunidades desiguais (GALTUNG, 1969). Pode-se identificar as vítimas, mas os agressores estão diluídos por trás das estruturas sociopolíticas, econômicas e culturais desiguais, que produzem, por exemplo, desempregados, vítimas de crises financeiras, vítimas da fome, da violência policial ou do sistema patriarcal.

Já a terceira concepção de violência seria a mais sutil e silenciosa de todas elas, a violência cultural ou cultura de violência, na qual não podemos identificar claramente nem vítimas nem agressores, uma vez que ela está presente nos discursos sociais, nos produtos culturais e comportamentos socialmente apreendidos, legitimando as duas outras tipologias. Isto significa que, por trás de um ato de violência direta, como um conflito armado, por exemplo, há fatores culturais que justificam, legitimam e naturalizam esse acontecimento. 0 termo violência cultural é análogo ao conceito de violência simbólica, definido pelo sociólogo francês Pierre Bourdieu. Segundo o autor, a violência simbólica é tácita: quem a sofre e a exerce 0 faz inconscientemente suas ações são "formas de coerção apoiadas na harmonia não consciente entre as estruturas objetivas e mentais" (BOURDIEU, 2014, p. 151).

Nesse contexto, a comunicação tem um papel fundamental na reprodução e propagação de estruturas de violência cultural, uma vez que naturaliza determinadas ideias, crenças, preconceitos, ideologias e comportamentos.

Da mesma maneira com que a violência trespassa a ponta do iceberg de forma silenciosa e devastadora, atingindo os seus níveis mais profundos, a paz pode ter características semelhantes: ela pode ser ensinada e aprendida, até que se intrique na cultura e crie nela longas raízes.

A transição da cultura de violência para uma cultura de paz "requer um esforço educativo prolongado para modificar as reações à adversidade e construir um modelo de desenvolvimento que possa suprimir as causas de conflito" (NOLETO, 2010, p. 12). Para Boulding (2002), cultura de paz pode ser definida como um mosaico de identidades, atitudes, valores, crenças e padrões que levam as pessoas a se relacionarem umas com as outras sem uma estrutura de poder, lidando criativamente com as diferenças e compartilhando seus recursos.

Muito além de definir o que é a cultura de paz em oposição à cultura de violência, é preciso, primeiramente, abordar quais são as 
implicações que a palavra paz carrega consigo. Uma definição amplamente difundida deste termo é a "ausência de guerras". Galtung (1984) traça um paralelo entre esta definição e a área da saúde: nas ciências médicas, o conceito da palavra saúde vai além de "ausência de doenças". Ele abrange, também, a manutenção de um corpo saudável, capaz de resistir a doenças. Correspondentemente, 0 autor surge com o conceito de paz positiva em oposição à paz negativa esta última, sim, como sendo ausência de guerras. A paz positiva compreende não somente os entremeios dos conflitos; ela é construída sobre os ideais de equilíbrio, cooperação e integração.

Wallensteen (2011) comenta que a tarefa de colocar a paz na agenda acadêmica internacional foi árdua: partes conflitantes e diversas instituições reclamaram esse objeto de estudos como de seu próprio domínio, enquanto outros 0 consideravam apenas um ramo dos estudos sobre segurança, defesa ou áreas correlatas. A fim de ampliar os conceitos modernos sobre a paz e investigá-los como métodos para a compreensão e transformação de conflitos, surgem os Peace Studies, ou estudos para a paz.

Os estudos para a paz são considerados uma área das ciências sociais aplicadas e têm como objeto de estudo a exploração científica da paz, visando à redução da violência entre os seres humanos na sociedade (CIANCIO; NOS ALDÁS, 2000). Esse é um objeto que não se gera pela não-ação; ele não é sólido e imutável, mas um estado e uma cultura, e deve ser buscado em âmbito social e pessoal (GONZÁLEZ; LÓPEZ, 2012).

A formalização da pesquisa da paz "parte de interrogações radicais sobre as causas profundas do conflito e sobre a totalidade dos passos necessários à sua superação", 0 que inclui uma ampla reestruturação social (PUREZA, 2000, p. 38). Com os estudos da guerra já consolidados e difundidos, pesquisadores que buscavam ensinar a paz e colocá-la à frente de suas investigações enfrentavam a marginalização, pois iam contra a corrente hegemônica das práticas acadêmicas (WEBEL, 2007). Por este motivo, aqueles que decidiram prosseguir com os estudos da paz criaram as suas próprias contra-instituições como redutos, nas quais não correriam o risco de isolamento profissional e acadêmico.

Stephenson (1989 apud MILLER, 2005) sumariza a evolução dos estudos para a paz em três diferentes etapas. A primeira tem início em 1930, liderada primariamente por acadêmicos e estudos com enfoque nas guerras como fenômeno social (polemologia). A segunda fase se inicia nos anos 1960, quando emergem os estudos de Johan Galtung ao fundar o Peace Institute Research em Oslo, na Noruega. A partir desta década, os estudos tornam-se mais amplos, passando a abranger 0 impacto das diferentes tipologias da violência e da injustiça, e difundidos, fazendo parte de grades curriculares de cursos ao redor do mundo. Na terceira fase, iniciada nos anos 
1980, movimentos sociais e organizações passam a inserir os estudos em suas ações. Martínez Guzmán (2009 apud SANDOVAL FORERO, 2012) adiciona uma quarta etapa ao histórico, iniciada nos anos 1990. Neste período, os estudos para a paz adotam uma perspectiva "holística, integral e completa", e pautam-se na diversidade como meio de preservação da paz, nas relações interculturais e no feminismo (Ibid., p. 24).

Tuvilla (2014), por sua vez, classifica a evolução dos estudos para a paz em três correntes: minimalista, intermediária e crítica ${ }^{2}$. Na primeira, a paz era relacionada à ausência de guerras internacionais. Esta corrente ignorava os conflitos internos e não questionava os interesses sociais, políticos e econômicos por trás das guerras; a segunda corrente já não considerava a paz como somente a ausência de guerras, mas sim como a ausência de quaisquer sistemas de ameaças, tanto a nível interno, quanto externo. Excluía-se, entretanto, os conceitos de violência estrutural e cultural; a corrente crítica incorpora os conceitos dos Peace Studies de Galtung, e suas investigações enfocam em todos os aspectos da violência e na paz positiva.

Logo nos anos 1970, pouco tempo após os primeiros estudos de Galtung, os estudos para a paz veem uma mudança de caráter epistemológico significativa: a "velha agenda" dos estudos, que investigava, por exemplo, as causas da guerra e as dinâmicas armamentistas, é contestada por uma "nova agenda" "que propunha debruçar-se sobre a exploração, a dominação e a dependência, o imperialismo etc." (WIBERG, 2005, p. 24). Este embate levanta críticas, por parte dos pesquisadores da nova agenda, de que os Peace Studies, até então, não tinham caráter emancipatório, e suas pesquisas "[situavam-se] prioritariamente numa lógica de tecnicidade e de procedimentalismo", em vez de estarem no terreno da teoria crítica (PUREZA, 2005, p. 3).

No Brasil, os estudos para a paz ainda se encontram em fase inicial. R. P. Costa (2009), em sua tese de doutorado, constatou que a produção de conhecimento científico dos estudos para a paz em âmbito brasileiro é praticamente inexistente. A autora analisou, no banco de dados de teses e dissertações da CAPES (Coordenação de Aperfeiçoamento de Pessoal de Nível Superior), as publicações que abrangiam, de alguma maneira, estes estudos. Como resultado da pesquisa, constatou-se que apenas quatro teses e uma dissertação propunham estudos semelhantes às propostas dos estudos para a paz. Também como resultado desta pesquisa, a autora observa que, no Brasil, a área de conhecimento dos estudos de paz é pouco difundida, sendo "mais conhecida pela sociedade brasileira como cultura de paz" (COSTA, R. P., 2009, p. 67).

É comum que encontremos uma literatura ampla a respeito da violência, mas não sob a ótica dos 
Peace Studies. J. M. D. Costa (2009) comenta que, desde os anos 1960, após o surgimento do Instituto de Investigação para a Paz de 0slo (PRI0), muitos autores brasileiros se dedicaram a estudar a paz, com enfoque em temas como a segurança, militarismo e políticas externas. Entretanto, segundo o autor, os estudos para a paz no Brasil se mantêm marginalizados em programas de graduação e pós-graduação em relações internacionais, a maioria deles enfatizando os estudos estratégicos ou da segurança.

\section{A interdisciplinaridade dos Peace Studies: as relações com a comunicação}

Os estudos para a paz são, em si, além de multiculturais, multidisciplinares (MARTÍNEZ GUZMÁN, 2005; SANDOVAL FORER0, 2012) e também interdisciplinares, uma vez que se propõem a realizar interfaces entre disciplinas e áreas do conhecimento que permitam pensar a paz a partir de distintas perspectivas. No âmbito destes estudos,

[...] para estar alerta contra o perigo de imposição de uns saberes ou de umas culturas e submissão de outros, que infelizmente ocorre com demasiada frequência, necessitamos também da interdisciplinariedade e interculturalidade. $0 u$ seja, a interpelação recíproca entre disciplinas e culturas. Não é suficiente o reconhecimento da multiplicidade ou pluralidade. A investigação e os estudos para a paz têm que estar submetidos à riqueza das tensões, matizes, transformação positiva das contradições entre essas culturas e saberes plurais para reconstruir, a partir do reconhecimento e de forma dinâmica, aquelas ca- racterísticas que se vão configurando em cada momento, como indicadores de transformação pacífica das relações humanas que produzam guerras, marginalização, miséria ou exclusão (MARTíNEZ GUZMÁN, 2005, p. 44).

Para Martínez Guzmán e Muñoz (2004), a concomitância de diversas disciplinas é necessária para abordar todos os aspectos pelos quais a paz se manifesta, promovendo maior transcendência científica e cooperação com movimentos sociais. Assim, muitos autores centralizam seus estudos em áreas diretamente relacionadas com os estudos para a paz e suas amplas ramificações, como a filosofia para a paz e a educação para a paz.

Martínez Guzmán (2005) afirma que não existe simplesmente uma disciplina ou cultura que detêm todas as respostas para a transformação das relações humanas. Na mesma linha de pensamento, segundo Galtung (2006), é ideal que estes estudos sejam interdisciplinares e internacionais, com cada investigador se abrindo para a grande divergência de ângulos e opiniões, favorecendo a tolerância e 0 diálogo. 0 autor norueguês compara os Peace Studies aos estudos feministas:

[...] assim como os estudos sobre a mulher tornam as condições de supressão e libertação das mulheres visíveis, os estudos para a paz tornam a paz visível, compreensível e exequível. Nenhuma disciplina acadêmica tem o monopólio da paz, assim como nenhuma disciplina tem o monopólio das mulheres - mas todas têm algo para contribuir (GALTUNG, 2006, p. 17, tradução nossa). 
Portanto, por seu caráter multidisciplinar, todos os campos do conhecimento podem contribuir para 0 avanço das investigações sobre a paz. A comunicação não seria uma exceção: ela é parte inerente de todos os seres humanos e pode ser usada para infindáveis propósitos. A comunicação é uma ferramenta fundamental para a manutenção de uma guerra ou para seu fim e, assim sendo, estratégias comunicacionais podem - e devem ser utilizadas em contexto de conflitos.

De acordo com Muñoz (2012), as pessoas são, desde suas origens, comunicativas e conflituosas; a comunicação é um meio para gerir os conflitos.

[...] uma boa comunicação favorece as vias pacíficas e uma má comunicação pode provocar situações violentas. [...] [0] exercício de responsabilidade favorece também a construção de alternativas propícias à transformação das injustiças por meios pacíficos e a evitar 0 desenvolvimento da violência (MUÑOZ, 2012, p. 13, tradução nossa).

Os meios de comunicação são potentes agentes de socialização e geradores de cultura (GONZÁLEZ; LÓPEZ, 2012). 0 que é debatido socialmente é amplamente relacionado com o que é abordado pela agenda midiática: o que os meios escolhem transmitir torna-se, constantemente, o que é discutido pelas pessoas; entretanto, caso omitam informações, elas dificilmente farão parte do imaginário social e, consequentemente, não terão impacto cultural e social algum. A produção simbólica dos meios influencia o comportamento cotidiano e é, para Muñoz (2012), uma das principais ferramentas sociais de legitimação e difusão de ideias, de maneiras de interpretar 0 mundo e de cosmovisões.

Apesar da pluralidade de meios de comunicação que podemos observar atualmente, especialmente face à dinâmica permitida com a Internet, são poucos os que determinam como será a agenda. Em diversas ocasiões, os meios disseminam o que está de acordo com seus interesses econômicos e de poder, não dando espaço às minorias, a pequenas comunidades ou a interesses que divirjam dos seus. Em situações de conflitos, as organizações midiáticas podem contribuir para naturalizar a cultura de violência; da mesma maneira, os meios podem, também, ser usados para legitimar a cultura de paz (SADIG; GUTA, 2011). Pelo fato de a cultura de paz não ser amplamente difundida, suas mensagens e seus ideais não circulam facilmente pelo discurso midiático hegemônico propagado pelos canais tradicionais de comunicação (GONZÁLEZ, 2012) nem pelos canais alternativos. Bruck e Roach (1993) defendem que o uso de imagens de violência e crueldade gera benefícios comerciais. 0s autores exemplificam:

Fotografias de crianças morrendo de fome nas planícies desérticas da Eritreia ou de corpos deformados nos edifícios bombardeados em Beirute têm um valor informativo, mas também podem ser utilizadas de maneira sensacionalista como recortes breves e impactantes, com o objetivo de promover uma emissora de televisão na guerra de audiência contra seus concorrentes (BRUCK; ROACH, 1993, p. 90, tradução nossa). 
Em pesquisa de Espinar e Hernández (2012), ao analisar a cobertura jornalística de 15 conflitos armados distintos, constatou-se que existe um interesse desproporcionado pela violência e um desinteresse manifesto pela paz, já que somente 1,6\% do conteúdo analisado correspondeu com os ideais de transformação de conflitos e paz positiva. Para Vicente (2015), as estruturas sociais desiguais e exclusivas que afetam 0 ser humano em todas as dimensões de suas vidas foram reforçadas pela comunicação e sua abordagem da paz negativa. De acordo com Salinas (2014), esta é uma maneira dos grupos de poder garantir o seu controle social.

\begin{abstract}
A baixa sensibilidade à dor e ao sofrimento dos outros favorece 0 individualismo e a indiferença. A percepção do mundo de maneira negativa (um ambiente perigoso e miserável) gera pessimismo, desconfiança e medo, restringindo a participação da população no espaço coletivo. Desta maneira, a mudança e a transformação social se fazem pouco prováveis. Será frequente a ideia de que tudo está mal e de que nada se pode fazer (SALINAS, 2014, p. 58, tradução nossa).
\end{abstract}

Esta estratégia vai em direção oposta às funções sociais da comunicação propostas por Nos Aldás, Seguí-Cosme e Machota (2008). Para os autores, a comunicação para a paz busca cultivar relações pacíficas entre os seres humanos e favorecer formas responsáveis de ação social e discursos sociais de paz. Ela consiste em uma plataforma onde se mesclam os conceitos dos Peace Studies, da comunicação e, também, da resolução e gestão de conflitos (SADIG; GUTA, 2011). Sua abordagem respeita as diversidades locais e a pluralidade no processo de superação de conflitos, buscando ações que tenham a paz como finalidade (VICENTE, 2015). Procura-se, desta maneira, ressaltando o conceito de paz positiva sobre a negativa, tratar cada caso de conflito e violência com unicidade, de acordo com 0 ambiente cultural em que estão inseridos. Esta comunicação tenta ver os conflitos como necessários e positivos, visando a sua transformação, dinâmica, em vez de uma resolução estática.

Para Signates (2000, p. 2), "a ação comunicativa representa, em termos políticos, um pressuposto da democracia, por se realizar não fora, mas dentro da tensão entre 0 entendimento e 0 desentendimento, sendo o desentendimento um fator constitutivo da relação e 0 entendimento". A Resolução A/RES/53/243 de 1999 da Assembleia Geral das Nações Unidas ${ }^{3}$ explicita, em seu artigo $7^{\circ}$, a importância do papel informativo e educativo dos meios de comunicação na promoção de uma cultura de paz. Copello e Rojas (2008) concluem que a comunicação e a educação em comunicação assumem papel determinante no processo de construção de paz: as interações comunicacionais evidenciam as condutas conflitivas, ativam ou catalisam conflitos e conduzem a sua resolução, violenta ou pacífica.

A comunicação, dotada da construção de conhecimentos e representações sociais, 
abriga uma pluralidade de discursos geradora de embates, por isto há a necessidade de dar voz aos grupos mais diversos e propiciar 0 diálogo (FERNÁNDEZ; MIGUEL; SANTOLINO, 2011). Salinas (2014, p. 63) lista uma série de características pertencentes à comunicação voltada para a paz: 1. compreensão e reflexão acerca dos problemas estruturais; 2 . debate sobre as alternativas de mudança social com 0 intuito de transformar os problemas estruturais; 3 . reconhecimento das diversas opiniões, crenças, percepções ou culturas, favorecendo o diálogo e 0 entendimento mútuo; 4. fomento de uma cidadania ativa e ciente das injustiças, como a exclusão, e de que a mudança será bem-sucedida somente com a participação do coletivo.

0 pluralismo é [uma] das condições inevitáveis para a consecução dos valores da cultura de paz e para a existência de uma comunicação democrática; entretanto, sua ausência volta a ser [uma] das tônicas generalizadas no sistema dos meios, tanto públicos quanto privados. [...] a falta de pluralismo suprime a diversidade de vozes presentes na sociedade e marginaliza os posicionamentos minoritários, uma vez que freia a conciliação de interesses cidadãos, socioculturais, econômicos e políticos, aprofundando a perda da qualidade democrática (BECERRA, 2012, p. 74-75, tradução nossa).

Através de meios de comunicação voltados para a paz, fontes alternativas de informação são oferecidas à população, anulando ou mitigando discursos de ódio (ALI; MACHARIA, 2011). Estes meios têm potencial para conscientizar as pessoas, promover a transformação de conflitos e construir uma cultura de paz.

\section{0 jornalismo para a paz}

Estuda-se o jornalismo e sua relação com a paz por este estar amplamente permeado pela violência. 0 jornalismo de guerra possui uma literatura extensa, diferentemente do que vem a ser denominado Peace Journalism ou, como traduzido neste estudo, jornalismo para a paz.

A associação entre mídia e a cultura de violência é tão ampla que a comunicação pode funcionar como propagandista da guerra, atribuindo diversos critérios de noticiabilidade a qualquer acontecimento de teor bélico e relacionado a conflitos (LYNCH, 2010). De acordo com Carvalho, Freire e Vilar (2012), o que se tornou importante nas coberturas jornalísticas é um grande interesse pelo crime a moldes sensacionalistas. Como exemplo, os autores citam a forma com que agressores e criminosos são tratados na mídia brasileira: é conferido a eles o status de celebridade, estampando capas de revistas e cedendo diversas entrevistas. 0 s meios de comunicação têm como responsabilidade apresentar soluções para conflitos, o que raramente fazem, já que disseminam os ideais da cultura de violência "como uma resposta ao cotidiano social que busca combater a rotina" (CARVALHO; FREIRE; VILAR, 2012, p. 437).

Para Marín (2011), algumas das tendências que caracterizam os meios de comunicação em conflitos armados são os interesses políticos e econômicos das partes dominantes, a 
concentração de poder, a espetacularização das notícias e banalização da violência, que contribuem para a cultura de violência, e falhas nas coberturas de guerra, por não apresentarem rigor ${ }^{4}$, contextualização e contraste na escolha de fontes, características apontadas por Bernabé (2009 apud MARÍN, 2011).

Jornalismo para a paz é um conceito que surge como reivindicação de novos esquemas, práticas e rotinas profissionais que substituam os parâmetros atuais da comunicação (SALINAS, 2014). Outros conceitos, concernentes às áreas da comunicação e jornalismo voltados às melhorias das condições de vida das pessoas, também começam a ser desenvolvidos, como jornalismo social, jornalismo preventivo e jornalismo público.

Mesmo com a variedade de nomenclatura dos termos que relacionam o jornalismo ou comunicação e as suas funções sociais, dependendo de enfoques específicos ou pontos de vista de diferentes pesquisadores, os conceitos apresentados acima se entrelaçam, pois se referem à potencialidade dos discursos na promoção de diálogos e debates públicos (SALINAS, 2014), de maneira pacífica e integrada.

Para Annabel McGoldrick e Jake Lynch (2000), importantes pesquisadores da área, o jornalismo para a paz traça novas linhas de conexão entre jornalistas, fontes e as consequências das abordagens, utilizando a análise e transformação de conflitos para alcançar equilíbrio, igualdade e rigor em coberturas jornalísticas, isto é, ele não reporta somente assuntos relacionados à paz, mas aplica elementos dos estudos da paz e de conflitos ao cotidiano da profissão. Conteúdos jornalísticos podem ser avaliados adequadamente ao serem comparados com os resultados de investigações dos Peace Studies (LYNCH; McGOLDRICK, 2007).

0 termo e o conceito do Peace Journalism são desenvolvidos por Galtung durante os anos 1970, mas seu estudo se amplia e populariza entre profissionais da mídia e universidades nos anos 1990, principalmente (LYNCH; McGOLDRICK, 2007). 0 objetivo de seu surgimento é contraporse ao jornalismo de guerra, dominante, focado na vitória de uns ("nós") contra a derrota de outros ("eles"). Este processo, voltado para a guerra, não se concretiza por acaso, já que reportar é uma questão de escolha. Deste modo, é possível que editores e repórteres, visando ao desenvolvimento do jornalismo para a paz, façam as escolhas do quê e como reportar, de modo a criar oportunidades à sociedade de considerar e valorar a não-violência como resposta aos conflitos (LYNCH; McGOLDRICK, 2007). Sendo assim, preza-se, no jornalismo para a paz, pelos impactos sociais e a qualidade dos conteúdos em vez de interesses econômicos de empresas, já que muitos 
veículos de comunicação convencionais realizam uma cobertura superficial e descontextualizada de problemas, com o objetivo de controle social (SALINAS, 2014).

No jornalismo orientado para a guerra, apenas os conflitos em si e suas consequências visíveis, como o número de feridos ou mortos, as armas utilizadas ou os danos materiais causados, são considerados. Neste caso, pode-se notar uma desumanização da parte adversária

("inimiga"), alcançada através da exposição

de inverdades sobre eles e acobertamento dos erros "nossos". Este modelo jornalístico tem como foco a elite e considera a vitória e 0 cessar-fogo como sinônimo de paz.

Estas definições, e as suas comparações com o modelo do jornalismo para a paz, foram propostas por Johan Galtung e estão apresentadas em detalhes na Tabela 1.

Tabela 1: Comparações entre o jornalismo para a paz e o jornalismo de guerra.

\begin{tabular}{|c|c|}
\hline Jornalismo para a paz & Jornalismo de guerra \\
\hline $\begin{array}{l}\text { I. Orientado para a paz/conflito } \\
\text { - Explora a formação de conflitos; há partes, objetivos e } \\
\text { problemas múltiplos } \\
\text { - Todas as partes ganham } \\
\text { - Espaço e tempo abertos: causas e consequências em } \\
\text { qualquer lugar, inclusive na história e na cultura } \\
\text { - Apresenta os conflitos com transparência } \\
\text { - Dá voz a todas as partes, com empatia e entendimento } \\
\text { - Vê conflito e guerra como problemas e foca na } \\
\text { criatividade } \\
\text { - Humaniza todas as partes, especialmente quando há } \\
\text { armamentos } \\
\text { - É proativo: busca a prevenção antes que a violência e } \\
\text { guerra ocorram } \\
\text { - Foca nos efeitos invisíveis da violência: traumas, } \\
\text { danos à estrutura e à cultura }\end{array}$ & $\begin{array}{l}\text { I. Orientado para a guerra/violência } \\
\text { - Foca na arena de conflito; há duas partes e um } \\
\text { objetivo (vencer) } \\
\text { - Uma parte ganha, a outra perde } \\
\text { - Espaço e tempo fechados: causas e consequências se } \\
\text { restringem à arena, focando em quem atirou a primeira } \\
\text { pedra } \\
\text { - Apresenta a guerra de forma obscura/secreta } \\
\text { - "Nós contra eles"; voz somente para "nós" } \\
\text { - Vê "eles" como o problema e foca em quem prevalece } \\
\text { na guerra } \\
\text { - Desumaniza "eles", especialmente quando há } \\
\text { armamentos } \\
\text { - É reativo: espera atos violentos para reportar } \\
\text { - Foca nos efeitos visíveis da violência: número de } \\
\text { mortos, feridos e danos materiais }\end{array}$ \\
\hline $\begin{array}{l}\text { II. Orientado para a verdade } \\
\text { - Expõe as inverdades de todas as partes }\end{array}$ & $\begin{array}{l}\text { II. Orientado para a propaganda } \\
\text { - Expõe inverdades sobre "eles" e ajuda a encobrir as } \\
\text { "nossas" mentiras }\end{array}$ \\
\hline $\begin{array}{l}\text { III. Orientado para as pessoas } \\
\text { - Foca no sofrimento de todos e dá voz a mulheres, } \\
\text { crianças, idosos }\end{array}$ & $\begin{array}{l}\text { Ill. Orientado para as elites } \\
\text { - Foca no "nosso" sofrimento; tem homens da elite } \\
\text { como porta-vozes }\end{array}$ \\
\hline $\begin{array}{l}\text { IV. Orientado para soluções } \\
\text { - Paz = não-violência + criatividade } \\
\text { - Destaca iniciativas voltadas para a paz, a fim de, } \\
\text { também, prevenir outras guerras } \\
\text { - Foca na estrutura, na cultura e em uma sociedade } \\
\text { pacífica } \\
\text { - Resultado: resolução, reconstrução, reconciliação }\end{array}$ & $\begin{array}{l}\text { IV. Orientado para a vitória } \\
\text { - Paz = vitória + cessar-fogo } \\
\text { - Oculta as iniciativas de paz até que a vitória já tenha sido } \\
\text { conquistada } \\
\text { - Foca em tratados, instituições e em uma sociedade } \\
\text { controlada } \\
\text { - Parte para outras guerras e retorna à mesma caso hajam } \\
\text { questões pendentes }\end{array}$ \\
\hline
\end{tabular}


0 jornalismo tem a tendência de reportar somente a visão de fontes oficiais, eventos em vez de processos e dualismo em relação às partes envolvidas nos conflitos (maniqueísmo), como afirmam Lynch e McGoldrick (2007) e foi demonstrado na tabela acima. Por isto, os autores colocam o jornalismo de guerra como um tipo de enquadramento que omite que jornalistas conspirariam para influenciar o público a apoiar guerras, já que este tipo de convenção noticiosa construída acerca de conflitos, muito comum, levaria-nos a supervalorizar respostas violentas e menosprezar as não-violentas. Por outro lado, Hanitzsch (2004 apud HANITZSCH, 2007) coloca o enquadramento do jornalismo para a paz como socialmente responsável, capaz de contribuir no processo de peacemaking e peacekeeping .

Apesar de ser um campo pouco conhecido por pesquisadores e jornalistas, Xavier Giró (2016), em entrevista para o autor, afirma que o jornalismo para a paz é feito por diversos profissionais, mesmo que eles o desconheçam ou não o percebam:

Para estes profissionais, não se basta a análise do que está acontecendo no momento - 0 que é bom, caso feito com propriedade. Há um passo a mais: discutem propostas alternativas de como abordar a violência, de como frear a escalada de um conflito. A profundidade dessas discussões colabora (ou não) para suas soluções.
Giró (2016) recorda que, mesmo que um jornalista rotule suas ações profissionais como "jornalismo para a paz", a prática pode não ser desempenhada de maneira correta: "o Peace Journalism pode ser conduzido de maneira irregular também. Ele pode ser somente um rótulo. Não existe um modo preciso, mecânico de se fazer o jornalismo para a paz.

Este é um campo aberto, que deve ser explorado integralmente". Para o pesquisador, é crucial pensar em todos os seres humanos como seres humanos, e também que há diversas razões por trás da violência - "a violência nunca é gratuita".

Diversas razões explicitam por que o jornalismo tradicional não noticia os fatos de maneira contextualizada e humanizadora. Giró (2012) elucida essa premissa a partir de três casos distintos: primeiramente, os jornalistas dispõem de pouco tempo para investigar e apresentar as informações; 0 espaço disponível para transmitir as informações limita os profissionais, mesmo que estejam bem informados ${ }^{5}$; e a falta de formação adequada colabora para a desinformação. 0 jornalismo para a paz é um processo lento, pois trabalha para contextualizar e transformar conflitos. Ele, nas palavras de Giró (2012), não oferece notícias a cada minuto; seu desenvolvimento tenta desdramatizar, aliviar a tensão, encontrar certa calma, explorar a complexidade do conflito e de seus atores e ter

Frente ao imediatismo do jornalismo atual, que demanda por uma grande quantidade de notícias em um curto período de tempo, o espaço em jornais, rádios, emissoras de televisão, portais de notícia etc. para conteúdos noticiosos aprofundados e contextualizados torna-se restrito. 
em conta os valores de todas as partes envolvidas, e não somente de uma delas. Estes aspectos caminham na direção oposta do modelo de negócios do jornalismo hegemônico, das grandes empresas que visam, primariamente, ao lucro.

De fato, a respeito da desdramatização e do alívio da tensão que o jornalismo para a paz pretende, podemos estabelecer um paralelo com o conceito de fast thinking e slow thinking de Guy Claxton, psicólogo britânico, que se dedicou ao estudo dessas duas formas de pensamento. Segundo escreve Honoré apud Comins Mingol (2008, p. 167, tradução nossa):

0 primeiro é racional, analítico, linear e lógico. É 0 que fazemos sob pressão, quando o relógio faz tic tac; é a primeira maneira de pensar dos computadores, a maneira como funciona 0 lugar de trabalho moderno; este pensamento contribui com soluções claras para problemas bem definidos. Em contrapartida, o pensamento lento é intuitivo, distorcido, criativo. É o que fazemos quando a pressão desaparece e temos tempo para deixar que as ideias sejam pensadas a fogo lento e a seu próprio ritmo. Suas percepções são abundantes e sutis.

Assim, partindo desse ponto de vista, talvez a tensão possa, efetivamente, permitir certas respostas que possam gerar soluções claras. Entretanto, determinadas questões ou eventos, especialmente de natureza mais complexa, estrutural ou cultural, podem demandar mais tempo para o desenvolvimento de percepções, amadurecimento de ideias e reflexões mais sutis e profundas. Segundo Milán Kundera, explica Honoré (2005, p. 36 apud COMINS MINGOL,
2008, p. 168, tradução nossa): "Nossa época está obcecada pelo desejo de esquecer e, para realizálo, se entrega ao domínio da velocidade; acelera o ritmo para nos mostrar que já não deseja ser recordada, que está cansada de si mesma e quer apagar a minúscula e trêmula chama de memória”. Nessa perspectiva, o rebaixamento da tensão pode permitir, também, o não esquecimento, a memória testemunhal e a denúncia.

Características que são opostas às do jornalismo para a paz, apontadas por Giró (2012, p. 87-88), são: - a superficialidade: não somente das declarações de uns e outros, mas também ao limitar o relato aos níveis visíveis, sem aprofundar nas raízes das disputas; a reiteração da violência direta em detrimento da violência estrutural é uma variável da superficialidade;

- a simplificação: é dado maior destaque aos líderes; personalizam-se tanto os conflitos que as ações dos atores de base são apagadas;

- a demonização: 0 foco em atores do mal tem servido para justificar a guerra como principal recurso.

Fazer um jornalismo com enfoque na paz não significa que não se falará sobre as guerras. Giró (2016, n.p.) afirma que "se há guerra, deve-se falar sobre ela, mostrar o horror, os interesses que a motivam, mas também que há pessoas trabalhando em prol da paz".

0 jornalismo para a paz em uma estrutura democrática pode "incrementar a eficácia de programas de desenvolvimento, reduzir o desnível 
socio-econômico, a corrupção e a exploração, e, também, aumentar o respeito social e 0 autorrespeito pelos elementos mais frágeis da sociedade" (SHINAR, 2008). Assim, de acordo com Shinar (2007 apud KEEBLE; TULLOCH; ZOLLMANN, 2010), o intuito do jornalismo para a paz

[...] não é reportar apenas 'boas notícias', mas sim conceber um modo mais justo de cobrir conflitos quando comparado às coberturas usuais, além de sugerir possibilidades de aprimorar a performance profissional, fortalecer valores humanos, morais e éticos na mídia, ampliar seus horizontes profissionais e acadêmicos e, também, fornecer melhor serviço público por meio da mídia (p. 3 , tradução nossa).

Este modelo de jornalismo aplica valor noticioso às iniciativas que relacionam a paz com a superação da violência cultural ou estrutural (ESPINAR; HERNÁNDEZ, 2012). Para Shinar (2008, p. 43), o jornalismo para a paz é uma estratégia "que visa à melhoria das representações da mídia, da construção da realidade e da consciência crítica".

A área do jornalismo para a paz recebe, constantemente, críticas a respeito da objetividade jornalística. 0 jornalismo, não importa sua especialidade ou por quem seja feito, baseia-se em escolhas (de enquadramentos, de fontes, de linguagem, de fotografias etc.). Não se nega que o Peace Journalism tome partidos, mas quem 0 exerce 0 faz buscando a humanização e a compreensão. Na prática jornalística, há sempre um posicionamento por trás: pode ser a empresa, os lucros ou o combate ao sofrimento humano; 0 importante é informar o público (NOS ALDÁs, 2016). Para Xavier Giró (2016, n.p.), "não há problemas em entender qual é a direção das soluções mais justas para transformar os conflitos e reduzir o sofrimento. Se não o fizermos, agimos como uma biruta, que aponta para onde 0 vento soprar com mais intensidade".

Apesar de 0 termo receber diversas definições e ser incorporado por vários pesquisadores, avanços em sua formulação teórica e epistemológica ainda são necessários (SÁNCHEZ, 2011). Para a autora, busca-se encontrar, com o diálogo entre pesquisadores de disciplinas distintas, uma metodologia adequada para a aplicação dos conceitos do jornalismo para a paz na análise do discurso dos meios de comunicação.

\section{Possíveis aplicações}

As contraposições apontadas por Johan Galtung entre o jornalismo de guerra e o jornalismo para a paz, conforme apresentado anteriormente na Tabela 1, fornecem aporte para trabalhos com 0 Peace Journalism a partir de duas perspectivas distintas: uma voltada à práxis jornalística e outra de caráter metodológico.

Na primeira perspectiva, o jornalismo para a paz serve como diretriz para um fazer jornalístico mais completo e humanizado, no qual 0 profissional se basearia nas características fundamentais do Peace Journalism durante a produção de conteúdos noticiosos. Dessa 
maneira, o jornalismo para a paz pode ser incorporado durante todas as fases do processo de reportagem: 0 jornalista pode optar, logo na produção da pauta e apuração, por um enquadramento que traga elementos do jornalismo para a paz (que aborde, por exemplo, em um contexto de conflito, a violência estrutural e cultural em vez da violência direta e que inclua, como fonte, pessoas diretamente afetadas por determinada situação, e não somente fontes institucionais). Na etapa de pós-produção, as características do jornalismo para a paz tornamse uma checklist pela qual o jornalista pode verificar se 0 material produzido é orientado para a paz positiva.

Um exemplo de iniciativa relacionada à práxis do jornalismo para a paz é a organização nãogovernamental libanesa Media Association for Peace (MAP) ${ }^{6}$. A ONG tem como missão a disseminação e advocacy do Peace Journalism. A partir de treinamentos de profissionais da comunicação e ativistas, workshops e projetos de reportagens, a MAP busca conscientizar sobre o papel fundamental da mídia em prol do peacebuilding e expandir os conhecimentos sobre o jornalismo para a paz em nível global. Algumas outras organizações que trabalham com o jornalismo para a paz são o Center for Global Peace Journalism, o Transcend Media Services e o Common Ground News Service (YOUNGBLOOD, 2017).
A outra perspectiva coloca o jornalismo para a paz como metodologia de pesquisa científica. Neste caso, o Peace Journalism é utilizado como parâmetro para a análise de conteúdos noticiosos e pode ser aliado a outros métodos, como a análise de conteúdo ou análise do discurso.

Um exemplo de como o jornalismo para a paz pode ser empregado como metodologia em pesquisas de comunicação foi proposto por Jake Lynch e Annabel McGoldrick (NICOLÁS GAVILÁN, 2013). Com o objetivo de investigar se as notícias promoveriam a paz ou exaltariam a violência em contexto de conflitos, os autores criaram um código de análise de notícias cujas categorias tiveram como base os conceitos do Peace Journalism. Foram criadas sete categorias, divididas em dois grupos: categorias de aproximação (contextualização; efeitos do conflito; orientação a pessoas comuns; e pluralidade de vozes) e categorias de linguagem (linguagem vitimizadora; linguagem que incita 0 medo; e emotividade).

A partir das categorias de Lynch e McGoldrick, Nicolás Gavilán (2013) conduziu um estudo de caso a fim de analisar os conteúdos noticiosos produzidos sobre 0 conflito Israel-Palestina por correspondentes internacionais para jornais espanhóis. A autora concluiu que mais da metade das notícias analisadas estavam contextualizadas e a cobertura foi equilibrada, no que tange a 
explorar os efeitos visíveis e invisíveis da violência - características do jornalismo para a paz.

\section{Considerações finais}

A ascensão ainda recente da investigação para a paz nos mostra que a busca pela paz é um processo lento e contínuo. A paz não se consolida a partir de ações pontuais, especialmente aquelas voltadas ao extermínio da guerra e dos conflitos aparentes. Os estudos para a paz propõem que se atinja a paz positiva mediante a incorporação de uma cultura voltada para a paz. Sugere-se, assim, que o enfoque seja dado aos conflitos que permeiam a cultura e as estruturas sociais.

Pela pluralidade da paz, os Peace Studies são comumente relacionados a outros campos do conhecimento. Eles já nascem com caráter multi e interdisciplinar e, em alguns contextos, até transdisciplinar, pois defendem que a paz pode ser alcançada a partir de iniciativas em distintas disciplinas: vemos que ganham força áreas como a educação para a paz, a filosofia para a paz e a comunicação para a paz, por exemplo, muitas vezes estabelecendo interfaces e incorporando diferentes olhares e perspectivas a fim de evidenciar uma ciência engajada e comprometida com a transformação social.

0 jornalismo para a paz pode servir como um tipo de manual de auxílio na cobertura jornalística, especialmente quando envolve guerras, conflitos e situações de violência. Coberturas jornalísticas consistem em escolhas (em seu enquadramento, em relação a fontes, à linguagem ou fotografias a serem utilizadas, etc.), e as escolhas feitas pelo jornalismo para a paz buscam a humanização e 0 entendimento, uma vez que dá voz a todas as partes, é orientado para as pessoas e para a cultura de paz em vez de somente reportar a violência direta. As coberturas devem ser equilibradas e atenção deve ser dada a todas as etapas do que se propõe a narrar: 0 antes (o que levou àquela situação), 0 durante (o que deve ser feito para transformá-la) e 0 depois (legado: quais são as consequências e estratégias de prevenção).

Observações sobre o jornalismo para a paz são importantes para que seja feito um "bom jornalismo". Este não é, claramente, a única maneira de ser fazer jornalismo. Veículos de comunicação e jornalistas podem desenvolver um jornalismo que siga os moldes do Peace Journalism sem que, ao menos, conheçam os seus conceitos. Além disso, podem haver outras estratégias para combater as injustiças e a violência por meio da comunicação. 0 jornalismo para a paz se trata, basicamente, de uma série de diretrizes eficazes para alcançar esses propósitos.

Além disso, conclui-se também que, em âmbito acadêmico, 0 jornalismo para a paz pode ser um grande aliado na análise de conteúdos noticiosos produzidos em contextos de violência. 0 Peace Journalism como metodologia pode fornecer uma nova perspectiva para os estudos do jornalismo. 


\section{Referências}

ALI, F. A.; MACHARIA, H. M. Peace media and the promotion of Culture of Peace and Peacebuilding: a case study of the Great Lakes Region in Africa. In: NOS ALDÁS, E.; GALÁN, J. I. M.; ALI, F. A. (Orgs.). Comunicación para la paz em acción: periodismos, conflictos, alfabetización mediática y Alianza de Civilizaciones. Castellón de la Plana, Universitat Jaume I, 2011. p. 159-174.

BECERRA, M. D. Problemas en la comunicación de las organizaciones sociales que trabajan por la cultura de paz. In: GONZÁLEZ, A.C.; LÓPEZ, M. C. (Orgs.). Comunicación y cultura de paz. Editorial Universidad de Granada: Granada, 2012. p. 69-82.

BOULDING, E. Peace culture. In: AFKHAMI, M. (Org.). Toward a compassionate society. WLP, 2002.

BOURDIEU, P. On the state: lectures at the Collège de France, 1989-1992. Cambridge: Polity Press, 2014.

BRUCK, P.; ROACH, C. Dealing with reality: the news media and the promotion of peace. In: ROACH, C. (Org.). Communication and culture in war and peace. Sage Publications, 1993. p. 71-96.

CARVALHO, D. W.; FREIRE, M T.; VILAR, G. Mídia e violência: um olhar sobre o Brasil. Revista Panamericana de Salud Pública, v. 31, n. 5, 2012, p. 435-438.

CIANCIO, A.; NOS ALDÁS, E. Comunicación para la paz y la cooperación: un proyecto conjunto. Jornades de Foment de la Investigació, 2000.

COMINS MINGOL, I. Filosofía del cuidar - una propuesta coeducativa para la paz. Barcelona: Icaria, 2008.

COPELLO, E. A.; ROJAS, L. R. Estudiantes: comunicación y cultura de paz. Revista Científica Electrónica de Ciencias Humanas, v. 11, n. 4, p. 28$65,2008$.

COSTA, J. M. D. Developing the Brazilian school for peace. Peace Review, v. 21, n. 1, p. 79-84, 2009.
COSTA, R. P. Multiculturalismo e estudos para a paz: articulação possível no reparo e no emprego de militares para missões de paz. 2009. 239 f. Tese (Doutorado em Educação) - Universidade Federal do Rio de Janeiro, Rio de Janeiro. 2009.

ESPINAR, E. R.; HERNÁNDEZ, M. I. S. El periodismo de paz como paradigma de comunicación para el cambio social: características, dimensiones y obstáculos.

Cuadernos de información y comunicación, v. 17, p. 175-189, 2012.

FERNÁNDEZ, A. V.; MIGUEL, J. C.; SANTOLINO, M. P. Comunicación para la paz en el sector de las 0NGs catalanas. Barcelona. 2008.

GALTUNG, J. Violence, peace, and peace research.

Journal of Peace Research., v. 6, n. 3, p. 167-191, 1969. Twenty five years of peace research: ten challenges, and some responses. Journal of Peace Research, v. 22, n. 2, 1984.

Violencia cultural. Gernika-Lumo: Gernika Gogoratuz, 2003.

Peace Studies: a ten point primer. In: HUNTER, A. (Org.). Peace Studies in the Chinese century. Ashgate, 2006. p. 15-20.

GIRÓ, X. Análisis crítico del discurso mediático y pedagogía comunicativa para la paz. In: GONZÁLEZ, A.C.; LÓPEZ, M. C. (Orgs.). Comunicación y cultura de paz. Editorial Universidad de Granada: Granada, 2012. p. 83-104.

Ideas sobre el periodismo para la paz.

Entrevista concedida a Jorge Salhani. Barcelona, maio 2016. n.p.

GONZÁLEZ, A. C. La comunicación es una herramienta estratégica en la construcción de paz. In: GONZÁLEZ, A. C.; LÓPEZ, M. C. (Eds.). Comunicación y cultura de paz. Editorial Universidad de Granada: Granada, 2012. p. 25-40.

GONZÁLEZ, A. C.; LÓPEZ, M. G. Introducción. In: GONZÁLEZ, A. C.; LÓPEZ, M. C. (Orgs.). 
Comunicación y cultura de paz. Editorial Universidad de Granada: Granada, 2012. p. 15-23.

HANITZSCH, T. Situating peace journalism in journalism studies: a critical appraisal. Conflict \& Communication, v. 6, n. 2, 2007.

KEEBLE, R. L.; TULLOCH, J.; ZOLLMANN, F. Peace Journalism, war and conflict resolution. Nova York: Peter Lang, 2010.

LYNCH, J. Propaganda, war, peace and the media. In: KEEBLE, R. L.; TULLOCH.; ZOLLMANN, F. (Orgs.).

Peace Journalism, war and conflict resolution. Nova York: Peter Lang, 2010. p. 69-83.

LYNCH, J.; McGOLDRICK, A. Peace Journalism. In: WEBEL, C.; GALTUNG, J. (Orgs.). Handbook of Peace and Conflict Studies. Nova York: Routledge, 2007. p. 248-264.

MARÍN, A. P. La verdade como víctima. Medios de comunicación como actores de guerra. In: NOS ALDÁS, E.; GALÁN, J. I. M.; ALI, F. A. (Orgs.). Comunicación para la paz em acción: periodismos, conflictos, alfabetización mediática y Alianza de Civilizaciones. Castellón de la Plana, Universitat Jaume I, 2011. p. 25-44.

MARTÍNEZ GUZMÁN, V. Filosofia e investigação para a paz. Revista Crítica das Ciências Sociais, v. 71, p. 43-62, 2005.

MARTÍNEZ GUZMÁN, V.; MUÑOZ, F. A. Investigación para la Paz. In: MARTÍNEZ, M. L. (Dir.) Enciclopedia de Paz y Conflictos: A-K. Granada: Editorial Universidade de Granada, 2004.

McGOLDRICK, A.; LYNCH, J. Peace Journalism

- What is it? How to do it? 2000. Disponível em:

< http://bit.ly/2kCWtdY>. Acesso em 25 mar. 2017.

MILLER, C. A. A glossary of terms and concepts in Peace and Conflict Studies. San Jose: University for Peace, 2005.

MUÑOZ, F. A. Prólogo. In: GONZÁLEZ, A. C.; LÓPEZ, M. C. (Orgs.). Comunicación y cultura de paz. Editorial
Universidad de Granada: Granada, 2012. p. 9-14.

NICOLÁS GAVILÁN, M. T. El conflicto israelípalestino en la mira. Análisis de las noticias de los corresponsales de prensa española (2007-2008) desde la perspectiva del Peace Journalism. Frontera Norte, v. 25, n. 50, p.. 65-95, 2013.

NOLETO, M. J. A construção da cultura de paz: dez anos de história. In: Cultura de paz: da reflexão à ação. Brasília: UNESC0; São Paulo: Associação Palas Athena, 2010. p. 11-22.

NOS ALDÁS, E.; SEGUÍ-COSME, S.; MACHOTA, A. M. R. Comunicación y construcción de paz. Barcelona: Icaria Editorial, 2008.

NOS ALDÁS, E. IUDESP y la comunicación para la paz. Entrevista concedida a Jorge Salhani. Castellón de la Plana, maio 2016.

PUREZA, J. M. Estudos sobre a paz e cultura de paz.

Nação e defesa, n. 95/96, p. 33-42, 2000. Os estudos para a paz - tendências e debates.

Revista Crítica de Ciências Sociais, v. 71, p. 3-4, 2005.

SADIG, H. B.; GUTA, H. A. Peace communication in Sudan: toward infusing a new Islamic perspective. In: FORTNER, R. S.; FACKLER, P. M. The handbook of global communication and media ethics. WileyBlackwell, 2011. p. 602-625.

SALINAS, A. I. A. Periodismo y comunicación para la paz. Indicadores y marco regulatório. Revista Comunicación y Ciudadanía Digital - COMMONS, v. 3, n. 1, p. 57-92, 2014.

SÁNCHEZ, M. H. Introducción. In: NOS ALDÁS, E.; GALÁN, J. I. M.; ALI, F. A. (Orgs.). Comunicación para la paz em acción: periodismos, conflictos, alfabetización mediática y Alianza de Civilizaciones. Castellón de la Plana, Universitat Jaume I, 2011. p. 81-86. SANDOVAL FORER0, E. A. Estudios para la paz, la interculturalidad y la democracia. Ra-Ximhai, v. 8, n. 2, p. 17-37, 2012. 
SHINAR, D. Mídia democrática e jornalismo voltado para a paz. Líbero, ano XI, n. 21, p. 39-48, 2008.

SIGNATES, L. Comunicação e Paz. Disponível em: $<$ http://bit.ly/2kbF5f9>. Acesso em 25 mar. 2017.

TUVILLA, J. Culturas de paz: fundamentos y claves educativas. Desclée de Brouwer: Bilbao, 2014.

VALESAN, S. Apresentação. In: WAISELFISZ, J. J.

Mapa da Violência: mortes matadas por armas de fogo. Brasília: 2015. Disponível em: < http://bit. ly/1h5RXOX>. Acesso em 23 mar. 2017.

VICENTE, M. M. Comunicación y paz activa: un diálogo posible. Intercom - Revista Brasileira de Ciências da Comunicação, v. 38, n. 1, p. 275-292, 2015.

WALLENSTEEN, P. Making peace researchable. In: WALLENSTEEN, P. (Org.). Peace research. Routledge. 2011. p. 3-12.

WEBEL, C. Introduction: toward a philosophy and metapsychology of peace. In: GALTUNG, J.; WEBEL, C. (Orgs.) Handbook of peace and conflict studies. Nova York: Routledge, 2007. p. 3-13.

WIBERG, H. Investigação para a paz: passado, presente e futuro. Revista Crítica de Ciências Sociais, v. 71, p. 21-42, 2005.

YOUNGBLOOD, S. Peace Journalism principles and practices: responsibly reporting conflicts, reconciliation, and solutions. Nova York: Routledge, 2017. 


\begin{tabular}{|l|}
\hline $\begin{array}{l}\text { Peace Journalism: } \\
\text { Concepts and reflections }\end{array}$ \\
\hline $\begin{array}{l}\text { Abstract } \\
\text { The culture of violence is legitimized as it } \\
\text { predominates in people's speech and in cultural } \\
\text { products. In this context, Peace Studies emerge from } \\
\text { a multidisciplinary and interdisciplinary perspective, } \\
\text { aiming to put in evidence the structures of violence, } \\
\text { to provide alternatives for the construction of a } \\
\text { culture of peace and for the consolidation of positive } \\
\text { peace. From the Peace Studies perspective, this } \\
\text { article presents Peace Journalism as an alternative } \\
\text { for journalistic practice and theories. Through } \\
\text { bibliographical and exploratory research method, } \\
\text { we think over the viability of Peace Journalism and } \\
\text { present two different approaches: a practical and } \\
\text { professional one, and a methodological one. } \\
\text { Keywords } \\
\text { Culture of Peace. Peace. Peace Journalism. Violence. }\end{array}$ \\
\hline
\end{tabular}

\section{Periodismo para la paz: conceptos y reflexiones}

\section{Resumen}

La violencia cultural es legitimada cuando es la que predomina en los discursos sociales y en los productos culturales. En este contexto, la investigación para la paz (Peace Studies) surge desde una perspectiva multi e interdisciplinaria con el objetivo de visibilizar las estructuras de la violencia, proponer alternativas para la construcción de una cultura de paz y para la consolidación de la paz positiva. Este artículo, bajo la mirada de la investigación para la paz, presenta la perspectiva del periodismo para la paz (Peace Journalism) como reivindicación de un nuevo hacer periodístico. A través de investigación bibliográfica y exploratoria, reflexionamos sobre la viabilidad del periodismo para la paz y presentamos dos abordajes distintos para su utilización: la práctica y profesional, y la metodológica.

\section{Palabras-clave}

Cultura de paz. Periodismo para la paz. Paz. Violencia. 


\section{Expediente}

A revista E-Compós é a publicação científica em formato eletrônico da Associação Nacional dos Programas de Pós-Graduação em Comunicação (Compós). Lançada em 2004, tem como principal finalidade difundir a produção acadêmica de pesquisadores da área de Comunicação, inseridos em instituições do Brasil e do exterior.

\section{E-COMPÓS I www.e-compos.org.br I E-ISSN 1808-2599}

Revista da Associação Nacional dos Programas de Pós-Graduação em Comunicação. Brasília, v.20, n.3, set./dez. 2017. A identificação das edições, a partir de 2008 , passa a ser volume anual com três números. Indexada por Latindex I www.latindex.unam.mx

\section{CONSELHO EDITORIAL}

Ada Cristina Machado Silveira, Universidade Federal de Santa Maria, Brasil Alda Cristina Silva da Costa, Universidade Federal do Pará, Brasil Alfredo Luiz Paes de Oliveira Suppia, Universidade Estadual de Campinas, Brasil Ana Regina Barros Rego Leal, Universidade Federal do Piauí, Brasil Ana Carolina Rocha Pessôa Temer, Universidade Federal de Goiás, Brasil André Luiz Martins Lemos, Universidade Federal da Bahia, Brasil Angela Cristina Salgueiro Marques, Universidade Federal de Minas Gerais, Brasil Ângela Freire Prysthon, Universidade Federal de Pernambuco, Brasil Antonio Carlos Hohlfeldt, Pontifícia Universidade Católica do Rio Grande do Sul, Brasil Arthur Ituassu, Pontifícia Universidade Católica do Rio de Janeiro, Brasil

Bruno Campanella, Universidade Federal Fluminense, Brasil

Cláudio Novaes Pinto Coelho, Faculdade Cásper Líbero, Brasil Cárlida Emerim, Universidade Federal de Santa Catarina, Brasil Carlos Eduardo Franciscato, Universidade Federal de Sergipe, Brasil Danilo Rothberg, Universidade Estadual Paulista, Brasil Denise Tavares da Silva, Universidade Federal Fluminense, Brasil Diógenes Lycarião, Universidade Federal do Ceará, Brasil Eduardo Vicente, Universidade de São Paulo, Brasil Eliza Bachega Casadei, Escola Superior de Propaganda e Marketing - SP, Brasil Eneus Trindade, Universidade de São Paulo, Brasil

Erick Felinto de Oliveira, Universidade do Estado do Rio de Janeiro, Brasil Erly Vieira Júnior, Universidade Federal do Espírito Santo, Brasil Francisco de Assis, FIAM-FAAM Centro Universitário, Brasi

Francisco Elinaldo Teixeira, Universidade Estadual de Campinas, Brasil Francisco Gilson R. Pôrto Jr., Universidade Federal do Tocantins, Brasil Frederico de Mello Brandão Tavares, Universidade Federal de Ouro Preto, Brasil Gabriela Reinaldo, Universidade Federal do Ceará, Brasil Gilson Vieira Monteiro, Universidade Federal do Amazonas, Brasil Gustavo Daudt Fischer, Universidade do Vale do Rio dos Sinos, Brasil Itania Maria Mota Gomes, Universidade Federal da Bahia, Brasil Jiani Adriana Bonin, Universidade do Vale do Rio dos Sinos, Brasil José Afonso da Silva Junior, Universidade Federal de Pernambuco, Brasil José Luiz Aidar Prado, Pontifícia Universidade Católica de São Paulo, Brasi Josette Maria Monzani, Universidade Federal de São Carlos, Brasi Juçara Gorski Brittes, Universidade Federal de Ouro Preto, Brasil
Juliana Freire Gutmann, Universidade Federal da Bahia, Brasil Laura Loguercio Cánepa, Universidade Anhembi Morumbi, Brasi Leonel Azevedo de Aguiar, Pontifícia Universidade Católica do Rio de Janeiro, Brasil Letícia Cantarela Matheus, Universidade do Estado do Rio de Janeiro, Brasil Luciana Coutinho Souza, Universidade de Sorocaba, Brasil Maria Ataide Malcher, Universidade Federal do Pará, Brasil Maria Elisabete Antonioli, Escola Superior de Propaganda e Marketing - SP, Brasil Maria das Graças Pinto Coelho, Universidade Federal do Rio Grande do Norte, Brasil Marialva Carlos Barbosa, Universidade Federal do Rio de Janeiro, Brasil Marcel Vieira Barreto Silva, Universidade Federal da Paraíba, Brasil Marcia Tondato, Escola Superior de Propaganda e Marketing, Brasil Marli Santos, Universidade Metodista de São Paulo, Brasil

Márcio Souza Gonçalves, Universidade do Estado do Rio de Janeiro, Brasil Mauricio Mario Monteiro, Universidade Anhembi Morumbi, Brasil Mayka Castellano, Universidade Federal Fluminense, Brasil

Mozahir Salomão Bruck, Pontifícia Universidade Católica de Minas Gerais, Brasil Nisia Martins Rosario, Universidade Federal do Rio Grande do Sul, Brasil Paolo Demuru, Universidade Paulista, Brasil

Paula Melani Rocha, Universidade Estadual de Ponta Grossa, Brasil Potiguara Mendes Silveira Jr, Universidade Federal de Juiz de Fora, Brasil Priscila Ferreira Perazzo, Universidade Municipal de São Caetano do Sul, Brasil Rafael Cardoso Sampaio, Universidade Federal do Paraná, Brasil Rafael Tassi Teixeira, Universidade Tuiuti do Paraná, Brasil Regiane Lucas Garcês, Universidade Federal de Minas Gerais, Brasil Regiane Regina Ribeiro, Universidade Federal do Paraná, Brasil Renata Pitombo Cidreira, Universidade Federal do Recôncavo da Bahia, Brasil Renato Essenfelder, Escola Superior de Propaganda e Marketing, Brasil Roberto Elísio dos Santos, Universidade Municipal de São Caetano do Sul, Brasil Rodolfo Rorato Londero, Universidade Estadual de Londrina, Brasil Roseli Figaro, Universidade de São Paulo, Brasil

Simone Maria Andrade Pereira de Sá, Universidade Federal Fluminense, Brasil Sofia Cavalcanti Zanforlin, Universidade Católica de Brasília, Brasil Sônia Caldas Pessoa, Universidade Federal de Minas Gerais, Brasil Tatiana Oliveira Siciliano, Pontifícia Universidade Católica do Rio de Janeiro, Brasil Thaïs de Mendonça Jorge, Universidade de Brasília, Brasil

Valquiria Michela John, Universidade Federal do Paraná, Brasil

\section{CONSELHO CIENTÍFICO}

Cristiane Freitas Gutfreind, Pontifícia Universidade Católica do Rio Grande do Sul, Brasil | Eduardo Antônio de Jesus, Universidade Federal de Minhas Gerais, Brasil I Eduardo Morettin, Universidade de São Paulo, Brasil I Irene de Araújo Machado, Universidade de São Paulo, Brasil I Miriam de Souza Rossini, Universidade Federal do Rio Grande do Sul, Brasil

\section{COMISSÃO EDITORIAL}

Eduardo Antonio de Jesus, Universidade Federal de Minas Gerais, Brasil I Igor Pinto Sacramento, Universidade Federal do Rio de Janeiro, Brasil I Kelly Cristina de Souza Prudencio, Universidade Federal do Paraná, Brasil I Osmar Gonçalves dos Reis Filho, Universidade Federal do Ceará, Brasil I Rafael Grohmann, FIAMFAAM - Centro Universitário, Brasil (editor associado)

\section{CONSULTORES AD HOC}

Afonso de Albuquerque, Universidade Federal Fluminense, Brasil I Francisco Rüdiger, Pontifícia Universidade Católica do Rio Grande do Sul, Brasil I Gislene da Silva, Universidade Federal de Santa Cataria, Brasil I Luiz Cláudio Martino Universidade de Brasília, Brasil I Magali Nascimento Cunha, Universidade Metodista de São Paulo, Brasil I Márcia Franz Amaral, Universidade Federal de Santa Maria, Brasil I Tania Marcia Cezar Hoff, Escola Superior de Propaganda e Marketing, Brasil I Raquel Paiva, Universidade Federal do Rio de Janeiro, Brasil

\section{EQUIPE TÉCNICA}

ASSISTENTE EDITORIAL Márcio Zanetti Negrini I REVISÃO DE TEXTOS Melina Santos I EDITORAÇÃO ELETRÔNICA Roka Estúdio
COMPÓS I www.compos.org.br

Associação Nacional dos Programas de Pós-Graduação em Comunicação

Presidente

Marco Roxo

Programa de Pós-Graduação em Comunicação - UFF marcos-roxo@uol.com.br

Vice-Presidente

Isaltina Gomes

Programa de Pós-Graduação em Comunicação - UFPE isaltina@gmail.com

Secretária-Geral

Gisela Castro

Programa de Pós-Graduação em Comunicação

e Práticas de Consumo - ESPM

castro.gisela@gmail.com

CONTATO I revistaecompos@gmail.com 